\title{
Evidence for a significant Blazar contamination in CMB anisotropy maps
}

\author{
P. Giommi ${ }^{1}$ and S. Colafrancesco ${ }^{2}$ \\ 1 ASI Science Data Center, ASDC, Agenzia Spaziale Italiana c/o ESRIN, via G. Galilei 00044 Frascati, Italy \\ 2 INAF - Osservatorio Astronomico di Roma via Frascati 33, 00040 Monteporzio, Italy \\ e-mail: cola@mporzio.astro.it
}

Received 11 June 2003 / Accepted 29 September 2003

\begin{abstract}
The analysis of the recent WMAP source catalog shows that the vast majority of bright foreground extragalactic sources detected in CMB maps are Blazars. In this paper we calculate the contamination of CMB anisotropy maps by this type of flat-spectrum, strongly variable and polarized extragalactic radio sources using up-to-date results from recent deep multifrequency surveys. From a careful archive search and from multi-frequency catalog cross-correlations we found that more than 50 known Blazars (or Blazar candidates) expected to be above the sensitivity limit of the BOOMERANG experiment are included in the $90 / 150 \mathrm{GHz}$ BOOMERANG anisotropy maps, a factor $\gtrsim 15$ larger than previously reported. Using a recent derivation of the Blazar radio $\log N-\log S$ we show that Blazars, whose counts continue to grow steeply down to faint fluxes, can sensitively affect CMB fluctuation maps with even moderate resolution and sensitivity. We calculate specifically that these sources induce an average sky brightness of $0.4 \mathrm{Jy} / \mathrm{deg}^{2}$, corresponding to an average temperature of $\approx 4-8 \mu \mathrm{K}$. Moreover, we find that the associated level of fluctuations is of the order of $C_{\ell, \text { Blazar }}=2 \times 10^{-2} \mu \mathrm{K}^{2}$ sr at $41 \mathrm{GHz}$ and $C_{\ell, \text { Blazar }}=1 \times 10^{-3} \mu \mathrm{K}^{2}$ sr at $94 \mathrm{GHz}$. Taking into account both Blazar variability, causing the detection of a number of weak sources that rise above the detector sensitivity during flares in long-exposure satellite experiments, and the many steep-spectrum radio sources that flatten at high frequencies, as well as the contribution of radio-galaxies, we find that the level of residual fluctuation due to discrete extragalactic foreground sources could be factor of $\sim 2-3$ higher than the above estimate. We show that the Blazar induced fluctuations contaminate the CMB spectrum at the level of $\approx 30-90 \%$ at $\ell=500$ and $80-100 \%$ at $\ell=800$, and thus they cannot be neglected in the derivation of the primordial CMB fluctuation spectrum. Since Blazars are bright sources because they point their jets towards the observer, at faint fluxes a more abundant population of less-aligned sources is likely to increase the contamination of future high-resolution high-sensitivity CMB maps. Careful cleaning for Blazar contamination of CMB maps is therefore necessary before firm conclusions about weak features, like secondary high- $\ell$ peaks of the CMB power spectrum or very weak signals like CMB polarization measurements, can be achieved.
\end{abstract}

Key words. cosmology: observations - cosmic microwave background - galaxies: active

\section{Introduction}

The Cosmic Microwave Background (hereafter CMB) contains - in nuce - a large amount of cosmological information. The paradigm follows from the fact that the CMB power spectrum is sensitive to the cosmological parameters which describe the fundamental properties of the universe (see, e.g., Jungman et al. 1996; Bond et al. 1997). The ability of the CMB data to constrain the cosmological parameters hinges upon the existence of acoustic oscillations in the primordial plasma at the last scattering epoch and on the ability of CMB experiments to disentangle such primordial fluctuations from those induced by cosmic structures at lower redshift.

CMB temperature fluctuations have been measured and studied in detail for the past decade starting with the

Send offprint requests to: P. Giommi, e-mail: paolo.giommi@asi.it large angular scale $(\theta \gtrsim 9$ deg corresponding to a scale $\ell \lesssim 20$ ) COBE detection (Smoot et al. 1992) up to the more recent and small-scale detection by BOOMERANG (De Bernardis et al. 2000), MAXIMA (Hanany et al. 2000), DASI (Halverson et al. 2002), VSA (Grainge et al. 2003), CBI (Pearson et al. 2003) and, lastly, by WMAP (Bennett et al. 2003a) which probes the CMB angular spectrum up to $\ell \sim 800$ over the whole sky with unprecedented precision. Extracting constraints on the cosmological parameters from the measurements of the CMB power spectrum is conceptually simple, but much more delicate in practice. In fact, the ability of the present and upcoming CMB experiments to determine the cosmological parameters requires a careful cleaning of the $\mathrm{CMB}$ maps from the galactic and extra-galactic foregrounds (see, e.g., Refregier \& Spergel 2000).

At high Galactic latitudes point-like sources are one of the main contaminants at small angular scales where, even when 
the brightest sources are identified and then subtracted, their residual contribution may dominate over the cosmic variance uncertainty. It has been shown, in fact, that point-like sources are one of the main foreground issue for the CBI experiment which is sensitive up to $\ell \sim 3500$ (Myers et al. 2003). However, such an issue also applies to other CMB experiments like VSA, DASI, or WMAP which probe the CMB angular spectrum at lower $\ell \lesssim 1000$ and it is nonetheless crucial for the up-coming space missions like PLANCK. Along this line of reasoning, Pierpaoli (2003) has recently shown that point-like sources induce a contamination of $\sim 20 \%$ level in the CMB power spectrum reconstruction even at $\ell \approx 200$ in the simulated WMAP map at $90 \mathrm{GHz}$. This level of contamination grows for increasing values of $\ell$ (i.e., for smaller angular scales) reaching a level of $\sim 80 \%$ at $\ell \approx 300$. This implies that point-like sources must be successfully identified and efficiently subtracted off the CMB maps before recovering the true primordial fluctuation spectrum. Pierpaoli (2003) also showed that bright sources have the greatest impact on the power spectrum at moderate $\ell$ values $(\ell \sim 400)$ while the fainter sources have a greater impact at high- $\ell$ values.

The recent WMAP experiment (Bennett et al. 2003a) resolved 208 bright pointlike sources, the vast majority of which are Blazars. We show in this paper that recent Blazar surveys, combined with detailed multi-frequency Spectral Energy Distribution analysis, provide evidence that a much larger number of Blazars and active galaxies must be present as positive temperature fluctuations already visible in the available CMB maps with even moderate spectral and spatial sensitivity. To this end, we discuss in Sect. 2 the astrophysical aspect of Blazars at radio and sub-mm frequencies and we discuss in Sect. 3 their contribution to the BOOMERANG 90/150 GHz anisotropy map. We also compare our results with the catalog of bright sources derived from the WMAP team. Based on an updated derivation of the Blazar counts, we derive in Sect. 4 the average sky brightness of the mm sky due to the Blazar population and we compute in Sect. 5 their angular power spectrum compared to the CMB power spectrum. We summarize our conclusions and we discuss the relevance of our results for the present and for the next coming CMB precision experiment in the final Sect. 6.

\section{Blazars at $\mathrm{cm}$ and $\mathrm{mm}$ wavelengths}

Blazars are a type of radio loud Active Galactic Nuclei (AGN) distinguished by their extreme, in some cases unique, properties. These include large amplitude rapid variability, apparent superluminal motion, high level of polarization and emission of radiation across the entire electromagnetic (e.m.) spectrum, from low frequency radio waves to gamma rays, up to $\mathrm{TeV}$ energies in some cases. This peculiar behaviour is thought to be due to physical phenomena observed under very special circumstances such as the emission of radiation from a relativistic jet of material ejected by a radio galaxy in the direction of the observer and seen at small angle with respect to the line of sight (Urry \& Padovani 1995; Blandford \& Rees 1978). The broad band e.m. spectrum of Blazars (represented in a $v f(v)$ vs. $v$ plane and usually referred to as the Spectral Energy
Distribution or SED) starts with a rising function of frequency (corresponding to a flat radio spectrum, $f(v) \sim$ constant, widely assumed to be synchrotron radiation) and increases with $v$ up to a maximum that is normally located at $10^{12}-10^{14} \mathrm{~Hz}$ but can also reach $\sim 10^{17} \mathrm{~Hz}$ in some extreme cases. Above the synchrotron peak the spectrum falls sharply until Inverse Compton (IC) emission emerges and rises again to form a second peak which is typically located at frequencies $v \approx 10^{17}-10^{19} \mathrm{~Hz}$ (see, e.g., Fig. 1 for two examples of Blazar SEDs; many other examples of Blazar SEDs can be found in the catalog of Giommi et al. 2002a).

Blazar are thought to be radio sources pointing their jet towards the observer, consequently they are rarer than other radio loud AGN. Since these sources emit strongly over the entire e.m. spectrum, while other types of AGN radiate mainly in the radio (up to a few $\mathrm{GHz}$ ), infra-red, optical, and X-ray band, Blazars -despite of their rareness- remain the extragalactic objects most frequently found in the Gamma-Ray, in the $\mathrm{TeV}$ and in the millimeter region.

In particular, Blazars are the dominant type of extragalactic sources in the microwave region of the e.m. spectrum where the CMB power peaks.

There are two types of Blazars: those showing an almost featureless spectrum, (that is with no broad emission lines) called BL Lacs, which are rare and do not show a strong cosmological evolution, and the Flat Radio Spectrum QSOs (FSRQ), which have broad lines in their optical spectrum, are much more abundant in flux limited surveys and evolve strongly on cosmological times (Urry \& Padovani 1995, Padovani 2002). FSRQs and BL Lacertae objects are thought to be the beamed counterparts of high and low luminosity radio galaxies respectively. Intensity and spectral variations are conspicuous at optical frequencies and become even larger at higher frequencies. Variability in the radio part of the e.m. spectrum, as demonstrated by a monitoring program lasting more than 25 years, can reach factors of $\sim 2$ to 10 on typical time-scales of weeks to years with a clear tendency to become more pronounced and more frequent at higher frequencies; polarization at radio frequencies is often present at a level of a few percent and in some case up to $10 \%$ (see, e.g., Hughes et al. 1992 and the on-line UMRAO database http://www . astro. lsa . umich. edu/obs/radiotel/umrao.html).

Radio sources of this type display a spectral shape which is not too different from that of the CMB spectrum before and around the peak of its power in the frequency range $\sim 50$ $200 \mathrm{GHz}$ (see, e.g., Fig. 1). For this reason Blazars can easily contaminate $\mathrm{CMB}$ maps (e.g., they can be confused with $\mathrm{CMB}$ fluctuations) if they are bright enough to be detected by CMB experiments. The recent results of the WMAP satellite experiment (Bennett et al. 2003a) clearly demonstrate that Blazars are present in large numbers in CMB fluctuation maps. In fact, we found, from a cross-correlation of the 208 sources of the WMAP catalog with catalogs of known sources and with the NED database, that $>87 \%$ of the WMAP sources are Blazarlike objects. Specifically, we found 140 FSRQ, 23 BL Lacs, 13 radio galaxies and 5 Steep Spectrum QSOs. Note that both radio galaxies and Steep Spectrum QSOs show Blazarlike characteristics: all of them are X-ray emitters, in most 
cases they are polarized and are associated to merging giant elliptical galaxies or $\mathrm{cD}$ galaxies in clusters. Six sources had no counterpart in any catalog probably because these are WMAP spurious detections, consistently with the expectations of Bennett et al. (2003b). The remaining 17 objects are still unidentified but may well be Blazars; in this case the percentage would grow to $98 \%$ !

Only 4 known sources (two planetary nebulas and two starburst galaxies) are associated with non-Blazar objets.

In addition to this evidence, we found that the three extragalactic sources detected well above the sensitivity threshold of the BOOMERANG maps (De Bernardis et al. 2000; Coble et al. 2003) are also Blazars and are included in the WMAP catalog.

To produce a more quantitative analysis of the contamination of CMB anisotropy maps by Blazars, we provide in the following, a list of the bright known Blazars which can be found in the 90/150 GHz BOOMERANG map. We choose to analyze the BOOMERANG maps because it covers a statistically representative large area of the sky $\sim 1800 \mathrm{deg}^{2}$ ) and because they are available (even though in a low resolution format) on the web (see, e.g., http://cmb. phys. cwru.edu/boomerang/).

\section{Blazars in the BOOMERANG CMB anisotropy maps}

We search here for Blazars that might have already been detected in the BOOMERANG maps but not yet recognized as such. To this aim, we have built the SED of all known Blazars for which that are enough published measurements (taken from NED and from various other surveys) to allow for a reasonably safe extrapolation of the flux intensity at the frequencies 90 and $150 \mathrm{GHz}$ of the BOOMERANG maps. We started our analysis from the few point-like sources that have already been recognized in the BOOMERANG maps (De Bernardis et al. 2000). Their follow-up (Coble et al. 2003) has shown their galactic (6 out of 9 sources) or extra-galactic ( 3 out of 9 sources) nature. The three extragalactic foreground sources are namely a Blazar (PKS 0537-441), a BL Lac (PKS 0521-365) and a Flat Spectrum Radio QSO (PKS 0438436). For one of these, namely PKS 0438-436, we report the SED in Fig. 1 where filled circles represent NED measurements and the three open diamonds at 90,150 and $240 \mathrm{GHz}$ represent the BOOMERANG measurements taken from Coble et al. (2003). To obtain a flux estimate around the peak of the CMB we fitted the multi-frequency points with a Synchrotron Self Compton (SSC) model (Perri et al. 2002, see also Perri 2003, Ph.D. Thesis, University of Rome, La Sapienza) which is a very good approximation of the SED of Blazars in the radio and $\mathrm{mm}$ band. The dotted and dashed-dotted curves in Fig. 1 represent the spectral distribution of typical CMB temperature fluctuations of the order of 300 and $50 \mu \mathrm{K}$ respectively, which can be clearly detectable in the BOOMERANG maps. Based on the SED shown in Fig. 1, the source PKS 0438-436 is then associated with a fluctuation of order $\gtrsim 300 \mu \mathrm{K}$, as already recognized in the BOOMERANG maps. The SED of the other sources PKS 0521-365 and PKS 0537-441 are similar to the previous case but are not reported here for the sake of brevity.
Following a similar procedure we found more than 50 Blazars, or Blazar candidates, selected through a multi-frequency technique like that used in the DXRBS (Perlman et al. 1998; Landt et al. 2001; Padovani 2002) using the NVSS and PMN, north and south of Dec $=-40$ deg respectively (radio), GSC2 (optical) and the Rosat (X-ray) all sky survey, for which the extrapolation of the radio data is above the detection limit of the BOOMERANG experiment. Since the SED of Blazars allows for fluxes above the BOOMERANG sensitivity and below the Rosat all sky survey sensitivity limit, the number of objects found is only a lower limit.

As an example of our findings, we show in the right panel of Fig. 1 the SED of the source PKS 0454-463 whose expected emission at $\mathrm{mm}$ frequencies is similar to that of the three Blazars already found in the BOOMERANG map. Whenever a Blazar was found to have a flux corresponding to a CMB fluctuation significantly above $50 \mu \mathrm{K}$, we have identified its position in the $150 \mathrm{GHz}$ BOOMERANG map.

We report in Fig. 2 the location of the whole set of Blazars found in the $150 \mathrm{GHz}$ BOOMERANG map. Their sky coordinates (RA and Dec), radio flux, optical $V$-band magnitude and redshift are also reported for completeness in Table 1.

To summarize, we found 54 known Blazars with flux greater than $\sim 200 \mathrm{mJy}$ that are probably associated with fluctuations of amplitude $\gtrsim 50 \mu \mathrm{K}$, in the $150 \mathrm{GHz}$ BOOMERANG map. The number of these Blazar point-like sources is a factor $>15$ larger than previously reported (Coble et al. 2003). A much larger number of fainter Blazars are evidently expected to be associated with lower amplitude $(\$ 50 \mu \mathrm{K})$ CMB fluctuations due to the fact that the Blazar counts steeply increase at flux fainter than $200 \mathrm{mJy}$, as will be discussed in the next Sect. 4.

The analysis of the BOOMERANG map is just an example of what we could find in a specific area of the sky surveyed at $\mathrm{mm}$ wavelengths. In particular, we estimate that more 200 Blazars should be found above the BOOMERANG sensitivity limit in $1000 \mathrm{deg}^{2}$ area. The WMAP anisotropy maps have basically the same sensitivity threshold and spatial resolution of the BOOMERANG maps and thus our results can be quite easily rescaled to the WMAP all-sky survey. Given the previous results, the Blazar populations are expected to produce a non-negligible contamination of the CMB maps as well as of the CMB power spectrum. We will address this point more specifically in the next Sects. 4 and 5.

\section{The sky brightness due to Blazars at $\mathrm{cm}$ and $\mathrm{mm}$ wavelengths}

Precise estimates of the contribution of Blazars to the sky brightness at radio and $\mathrm{mm}$ frequencies have so far been hampered by the lack of sufficiently deep surveys. For a long time only very shallow ( 1 or $2 \mathrm{Jy}$ ) surveys have been available at $\mathrm{cm}$ frequencies (Stickel et al. 1991; Wall \& Peacock 1985). However, the recent multi-frequency approach to Blazar search is changing the picture (e.g. Laurent-Muehleisen et al. 1998; Giommi et al. 1999; Padovani 2002; Giommi et al. 2002b,c), making it possible to detect many fainter Blazars. 

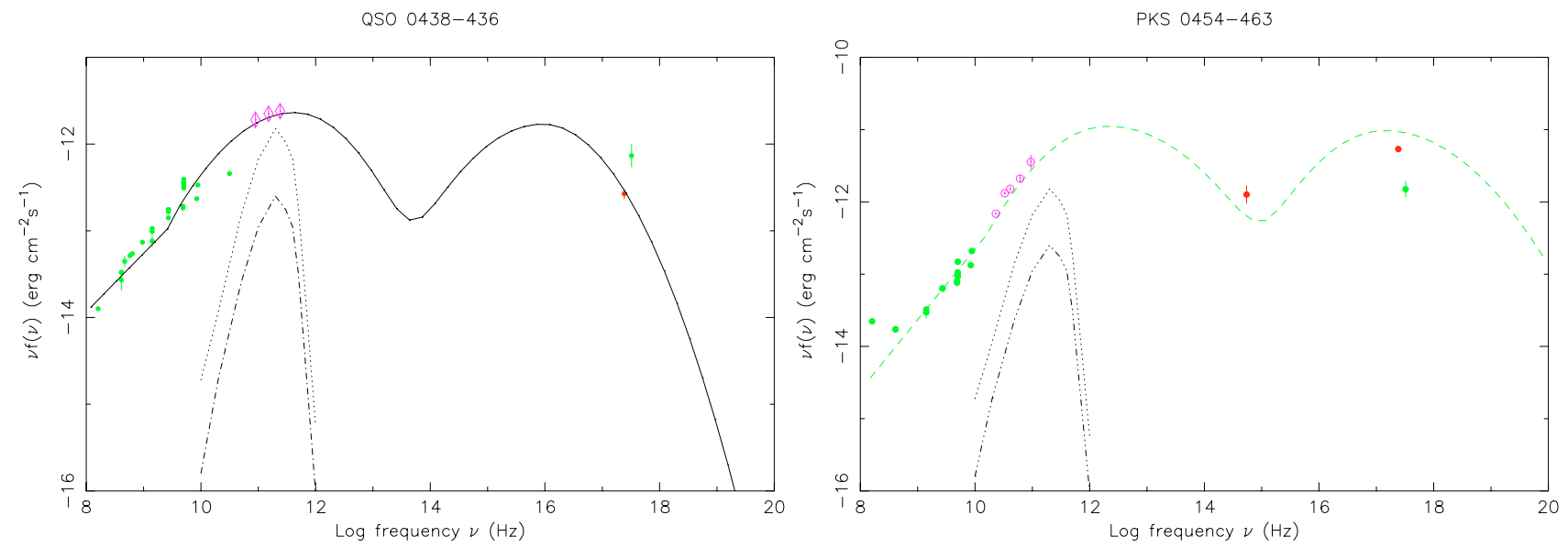

Fig. 1. Left: we show the SED of the FSRQ source, PKS 0438-436, one of the three Blazars reported by the original authors to have been detected in BOOMERANG maps (De Bernardis et al. 2000; Coble et al. 2003). The three open diamonds at 90,150 and $240 \mathrm{GHz}$ are, in fact, BOOMERANG measurements taken from Coble et al. (2003). The dotted and dashed-dotted curves below the data points, represent the frequency spectrum of CMB fluctuations of the order of 300 and $50 \mu \mathrm{K}$, respectively. The solid curve shows the SED in the $\log (v)-\log \left(v f_{v}\right)$ plane as expected from the Synchrotron Self Compton (SSC) model. Right: we show here the SED of the FSRQ source PKS 0454-463, whose expected flux at $\mathrm{mm}$ frequencies, estimated from the SSC model and shown as a dashed curve, is well above the BOOMERANG detection limit. This extrapolation is confirmed by the WMAP detection of this source at 23, 33, 41, 61 and $94 \mathrm{GHz}$ (Bennett et al. 2003b) which are shown as open points. The dotted and dashed-dotted curves below the data points, represent again the frequency spectrum of CMB fluctuations at the BOOMERANG resolution of the order of 300 and $50 \mu \mathrm{K}$, respectively.

The integrated radio flux due to a population of discrete sources in the flux range $S_{\min }-S_{\max }$ can be calculated as follows:

$I=\int_{S_{\min }}^{S_{\max }} S \frac{\mathrm{d} N}{\mathrm{~d} S} \mathrm{~d} S$

where the quantity $\mathrm{d} N / \mathrm{d} S$ is usually referred to as the differential $\log N-\log S$ distribution that, for a population of sources with constant density, is given by a power-law with slope equal to 2.5 which then flattens at faint fluxes of order of $\$ 1-10 \mathrm{mJy}$, depending on the Blazar luminosity function. The $\log N-\log S$ of BL Lacs is presently known down to $\approx 3.5 \mathrm{mJy}$ for the subsample of extreme High Energy Synchrotron peaked (HBL) sources (Giommi et al. 1999, 2002b) while the best estimate of the $\log N-\log S$ of the full population of BL Lacs and FSRQs currently comes from the Deep X-Ray Blazar Survey (DXRBS, Landt et al. 2001). This is the deepest complete and radio-flux limited Blazar survey presently available which extends to $50 \mathrm{mJy}$ at $5 \mathrm{GHz}$ (Padovani et al. 2003). At radio frequencies, the space density of FSRQs is higher than that of BL Lacs by about a factor of $\approx 4$ and show a strong cosmological evolution, while BL Lacs either do not evolve or show negative cosmological evolution (Padovani 2002; Giommi et al. 1999; Bade et al. 1998; Rector et al. 2000). The $\log N-\log S$ of BL Lacs is approximately Euclidean up to at least $50 \mathrm{mJy}$, while below $20 \mathrm{mJy}$ it flattens substantially, at least in the subsample of extreme HBL BL Lacs (Giommi et al. 2002b). From the DXRBS survey we know that the surface density of sources brighter than $0.1 \mathrm{Jy}$ at $5 \mathrm{GHz}$ is 0.18 object per square degree for FSRQs and 0.025 objects per square degree for BL Lacs (Giommi et al. 2002b; Padovani et al. 2003). We can then assume a Blazar overall density of $0.2 \mathrm{deg}^{-2}$ with flux $S \geq 0.1 \mathrm{Jy}$. Since FSRQs (the dominant Blazar population) evolve strongly, while BL Lacs show no evolution (or slightly positive) at high radio fluxes (Padovani 2002), and negative evolution below 20 mJy (Giommi et al. 1999, 2002b), we can conservatively assume an overall Blazar differential $\log N-\log S$ with a slope of 2.65 up to $50 \mathrm{mJy}$ below which it flattens significantly to a slope of $\sim 2.2$. This assumption is fully supported by the $\log N-$ $\log S$ (see Fig. 3) that we have built using a sample of 135 Blazars (BL Lacs + FSRQs) from the NVSS-RASS $1 \mathrm{Jy}$ and the 0.1 and 0.05 Jy points of the DXRBS (Padovani et al. 2003). While this $\log N-\log S$ is consistent with the counts of flat spectrum radio sources derived by Toffolatti et al. (1998) at $\approx 1 \mathrm{Jy}$, it does not show any flattening until a rather faint radio flux is reached and not just below $1 \mathrm{Jy}$ as in the predictions based on Toffolatti et al. (1998).

Thus, given the previously discussed $\log N-\log S$, and setting consistently the lower integration limit to $1 \mathrm{mJy}$, Eq. (1) yields a radio brightness of $\approx 0.4 \mathrm{Jy} / \mathrm{deg}^{2}$ at $5 \mathrm{GHz}$. In order to properly extrapolate this brightness to higher frequencies, we have to make use of the distribution of radio to $\mathrm{mm}$ spectral slopes for the Blazar population. To estimate realistic slope values we have built the Spectral Energy Distribution (SED) of all Blazars in the NVSS-RASS 1 Jy survey (Giommi et al. 2002c) using the many radio measurements available from the literature (mostly taken from NED). Since all objects in the NVSSRASS survey are brighter than $1 \mathrm{Jy}$, several radio data points are normally available for each Blazar over a wide frequency range. We have then fitted a power law to the radio part of spectrum and measured the distribution of spectral slopes $\alpha$ (here $f(v) \propto v^{-\alpha}$ ) between the $\mathrm{cm}($ e.g., $\approx 5 \mathrm{GHz}$ ) and $\mathrm{mm}$ region (e.g., $\approx 41,90,94$ and $150 \mathrm{GHz}$ ). We have derived the normalized distribution shown in Fig. 3 using the subsample of 135 objects with $\alpha<0.5$ which is a requirement normally applied to build Blazar samples. Using such a spectral 


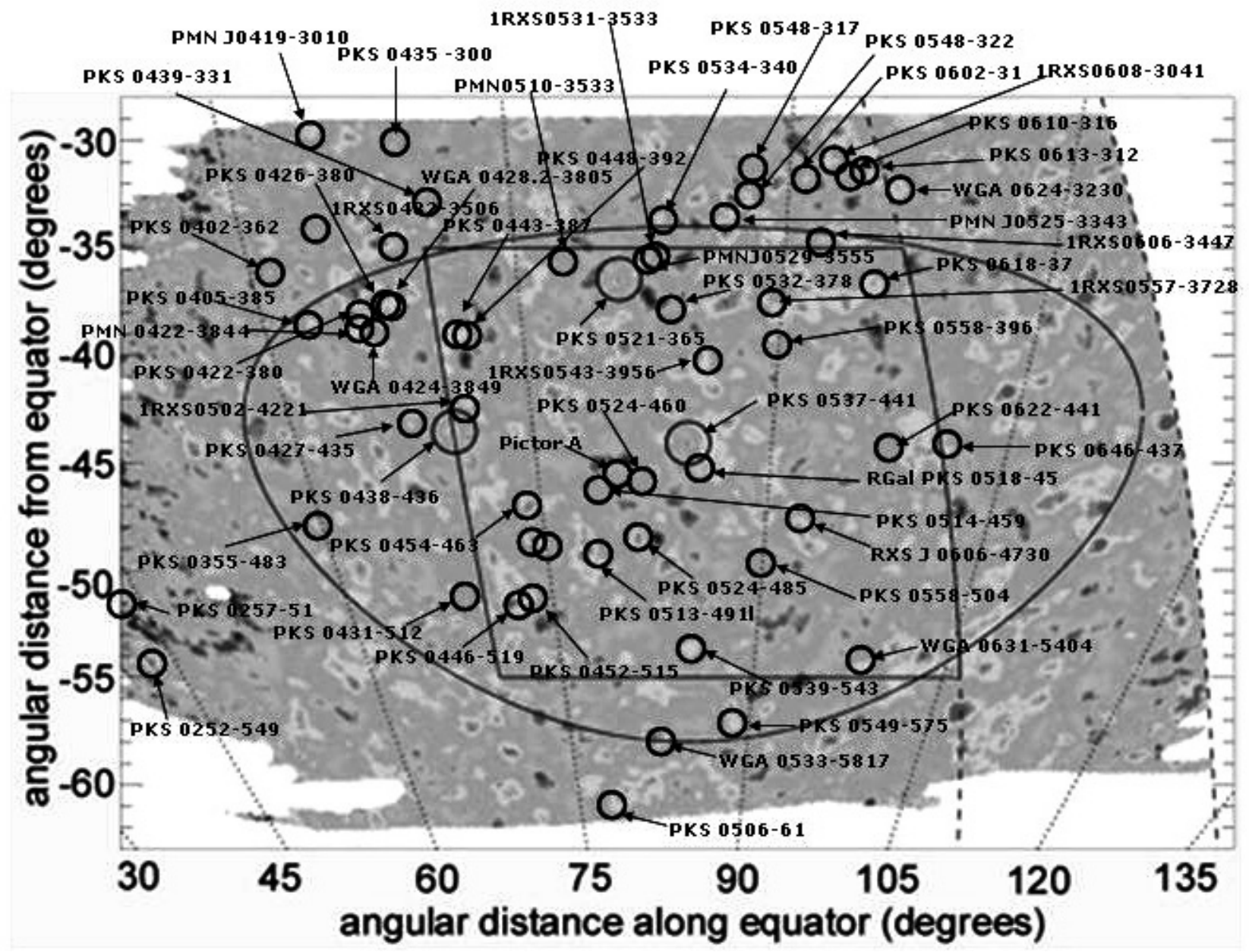

Fig. 2. The $150 \mathrm{GHz}$ CMB fluctuation map (taken from http://cmb.phys.cwru.edu/boomerang/) obtained from BOOMERANG is here correlated with the positions of known and candidate high galactic latitude $(|b| \gtrsim 20$ ) Blazars (and radio galaxies) whose expected flux at $150 \mathrm{GHz}$ (estimated from lower frequency archive data measurements) is high enough to be above the BOOMERANG detection limit, and should appear as green/yellow or red areas (see Table 1. See also http://www. asdc.asi.it/boomerang/ for the full list of FSRQ SEDs). Some of these objects (12 out of the 54 Blazars we list in Table 1) are also included in the WMAP source catalog.

slope distribution, the expected $150 \mathrm{GHz}$ sky brightness due to Blazars ranges from $\approx 3.6 \times 10^{10}$ to $\approx 7 \times 10^{10} \mathrm{Jy} \mathrm{Hz} \mathrm{deg}^{-2}$ depending mildly on the assumptions for the $\log N-\log S$ parameters. Since the $\mathrm{CMB}$ brightness at $150 \mathrm{GHz}$ is $\approx 2.1 \times$ $10^{16} \mathrm{Jy} \mathrm{Hz} \mathrm{deg}^{-2}$ (e.g. Hasinger 2000), the Blazars brightness is $\approx(2-3) \times 10^{-6}$ that of the $\mathrm{CMB}$, which is only a factor of a few below the level of the presently measured CMB fluctuations and similar to the sensitivity planned for the upcoming PLANCK mission. At 41 and $94 \mathrm{GHz}$ we obtain a sky brightness of $4-8 \mu \mathrm{K}$ assuming that the slope of the $\log N-\log S$ flattens from 2.65 to 2.2 at $50 \mathrm{mJy}$ and integrating it down to 1 and $0.1 \mathrm{mJy}$, respectively. This estimate is only a lower limit to the average sky brightness induced by Blazars since the DXRBS and NVSS-RASS 1 Jy surveys only include sources with flat radio spectrum between 1.4 and $5 \mathrm{GHz}$, while it is well known that a number of radio source spectra that are steep at low frequencies progressively flatten at higher frequencies. Using $15 \mathrm{GHz}$ source counts measurements Taylor et al. (2003) estimate that the contribution of steep-spectrum sources increase the extrapolated source counts at high frequency by a factor of $\sim 2$.
In addition, other sources that are not accounted for in Blazar surveys have been detected, as a minority population but still in fair numbers, in the WMAP experiment. These are, e.g., radio galaxies which have a often extended emission with steep radio spectrum, that outshines a much flatter spectrum nuclear component which may emerge and dominate the emission above a few tens of GHz. One example of such sources is Pictor A, a bright radio galaxy with extended steepspectrum emission and a flat-spectrum Blazar like, nuclear component (Fiocchi \& Grandi 2003, in preparation), which is included in the WMAP source catalog and is also present in the BOOMERANG maps (see Sect. 2).

\section{The angular power-spectrum contributed by Blazars}

Assuming that the Blazars are spatially distributed like a Poissonian random sample, then the angular power spectrum, $C_{\ell, \text { Blazar }}$, contributed by these sources is given by

$C_{\ell, \text { Blazar }}=\int_{S_{\min }}^{S_{\max }} \mathrm{d} S \frac{\mathrm{d} N}{\mathrm{~d} S} S^{2}$, 
Table 1. The list of flat-spectrum radio sources (mostly Blazars) found in the BOOMERANG maps. For each object we list the source name (Col. 1), the coordinates Ra (Col. 2) and Dec (Col. 3), the radio flux at $5 \mathrm{GHz}$ in units of mJy (Col. 4), the optical $V$-band magnitude (Col. 5), the redshift taken from NED (Col. 6). Some notes on the listed sources are added in Col. 7 of the table.

\begin{tabular}{|c|c|c|c|c|c|c|}
\hline Source name & ra(J2000.0) & $\overline{\mathrm{Dec}(\mathrm{J} 2000.0)}$ & Radio flux & 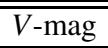 & Redshift & Notes \\
\hline PKS 0252-549 & 025330.7 & -544140.9 & 1193 & 17.7 & 0.537 & FSRQ - WMAP \\
\hline PKS 0257-510 & 025837.7 & -505215.9 & 452 & 23 & 0.834 & FSRQ \\
\hline PKS 0308-611 & 030955.6 & -605827.4 & 1103 & 18.6 & - & NED: QSO \\
\hline PKS 0310-558 & 031205.8 & -554138.0 & 501 & 18 & - & Blazar candidate \\
\hline PKS 0317-570 & 031855.9 & -565052.0 & 257 & 17.5 & - & Blazar Candidate \\
\hline PMN J0321-3711 & 032123.2 & -371133.0 & 5020 & - & - & NED: Radio S. Extended - WMAP \\
\hline PKS 0340-372 & 034205.4 & -370310.9 & 872 & 18.1 & 0.284 & QSO \\
\hline PKS 0402-362 & 040353.0 & -360447.9 & 1132 & 17.2 & 1.417 & FSRQ \\
\hline PKS 0405-385 & 040658.7 & -382622.9 & 830 & 17.7 & 1.285 & FSRQ - WMAP \\
\hline PKS 0410-519 & 041136.1 & -514919.9 & 361 & 17.5 & - & NED: QSO \\
\hline PMN J0419-3010 & 041948.2 & -301006.9 & 184 & 17.5 & - & Blazar candidate \\
\hline PMN J0422-3844 & 042214.6 & -384448.1 & 130 & 17 & 3.11 & NED: QSO \\
\hline WGA J0424.6-3849 & 042439.2 & -384901.9 & 309 & 18.5 & 2.34 & FSRQ \\
\hline PKS 0422-380 & 042441.8 & -375623.9 & 1706 & 18.1 & 0.782 & FSRQ - WMAP \\
\hline WGA J0428.8-3805 & 042850.4 & -380544.1 & 51 & 16.5 & 0.15 & BL Lac \\
\hline 1RXS J043208.7-35065 & 043208.6 & -350651.1 & 182 & 18 & - & Blazar candidate \\
\hline PKS 0432-606 & 043334.0 & -603014.0 & 636 & 19 & - & NED: QSO \\
\hline PKS 0435-300 & 043737.4 & -295411.1 & 691 & 17.2 & 1.328 & FSRQ \\
\hline $0438-43$ & 044017.7 & -433256.0 & 3933 & 18.8 & 2.852 & FSRQ - WMAP \\
\hline PKS 0439-331 & 044134.6 & -325952.0 & 197 & - & - & NED: Blazar candidate \\
\hline 1RXS J044510.0-38382 & 044510.0 & -383824.0 & 244 & 17 & - & NED: QSO - PKS 0443-387 \\
\hline PKS 0448-392 & 044942.0 & -391051.9 & 819 & 16.5 & 1.288 & FSRQ \\
\hline PKS0450-469 & 045152.5 & -465302.0 & 541 & 18.3 & - & NED: QSO \\
\hline 1Jy0454-463 & 045551.2 & -461601.9 & 1653 & 17.4 & 0.858 & FSRQ - WMAP \\
\hline PKS 0506-61 & 050643.6 & -610939.9 & 1211 & 16.9 & 1.093 & FSRQ - WMAP \\
\hline NGC 1808 & 050743.0 & -373029.8 & 238 & 12.6 & 0.003 & Starburst gal. \\
\hline PMN J0510-3533 & 051054.0 & -353309.0 & 123 & 18.1 & - & Blazar candidate \\
\hline PKS 0511-484 & 051251.4 & -482409.0 & 1094 & 19.8 & 0.306 & NED: Radio galaxy \\
\hline Fairall 790 & 051435.4 & -490333.1 & 310 & 15.9 & 0.09 & NED: Elliptical gal. \\
\hline 1Jy 0514-459 & 051548.1 & -455700.0 & 990 & 17.5 & 0.194 & FSRQ \\
\hline PKS 0518-45 & 051949.0 & -454645.8 & 14999 & 15.8 & 0.035 & FSRQ - WMAP \\
\hline PKS 0521-365 & 052259.4 & -362801.9 & 8180 & 14.6 & 0.055 & BL Lac - WMAP \\
\hline PMN J0525-3343 & 052504.9 & -334300.1 & 210 & 18.7 & 4.4 & FSRQ \\
\hline PMN J0529-3555 & 052937.1 & -355522.0 & 329 & 17.7 & - & Blazar Candidate \\
\hline 1RXS J053132.1-35333 & 053132.0 & -353330.9 & 173 & 18.5 & 0.9 & Blazar candidate \\
\hline PKS 0532-378 & 053418.0 & -374707.0 & 528 & 19.5 & 1.668 & NED: QSO \\
\hline PKS 0534-340 & 053628.5 & -340112.0 & 663 & 17.8 & 0.683 & FSRQ \\
\hline 1Jy0537-441 & 053851.0 & -440506.0 & 4805 & 15 & 0.896 & BL Lac - WMAP \\
\hline PKS 0539-543 & 054047.2 & -541820.8 & 373 & 18.2 & - & Blazar Candidate - WMAP \\
\hline 1RXS J054329.1-39563 & 054329.1 & -395638.0 & 174.8 & 17.9 & - & Blazar candidate \\
\hline PMNJ0545-4757 & 054506.4 & -475711.9 & 294 & 17 & - & Blazar Candidate in Cluster \\
\hline PKS 0549-575 & 055008.1 & -573230.8 & 355 & 19.5 & - & QSO from NED - WMAP \\
\hline PKS 0548-322 & 055040.7 & -321618.8 & 213 & 15.5 & 0.069 & BL Lac \\
\hline PKS 0548-317 & 055047.4 & -314359.8 & 1034.5 & 9.9 & 0.033 & BL Lac \\
\hline PKS 0555-374 & 055711.1 & -372813.0 & 152 & 14.2 & 0.0044 & Elliptical in Cluster \\
\hline 1RXS J060414.4-31560 & 060414.4 & -315602.0 & 2960.4 & 18.5 & 0.452 & FSRQ \\
\hline PMN J0606-3448 & 060608.1 & -344743.0 & 135 & 17.9 & 2.28 & NED: QSO \\
\hline PKS 0607-605 & 060755.0 & -603155.9 & 558 & 18.2 & - & NED: QSO \\
\hline PKS 0606-306 & 060840.9 & -304138.0 & 100 & 16.9 & - & Blazar Candidate \\
\hline PKS 0610-316 & 061229.2 & -313914.0 & 643 & 18.5 & - & NED: QSO \\
\hline PKS 0613-312 & 061516.8 & -311659.8 & 173.6 & 17 & - & NED: QSO \\
\hline PKS 0410-519 & 062202.1 & -473552.0 & 315 & 13 & - & Blazar Candidate \\
\hline WGA J0631.9-5404 & 063159.5 & -540431.0 & 155 & 18.2 & 0.193 & FSRQ \\
\hline PKS 0646-437 & 064816.7 & -434700.9 & 126 & 18 & 1.029 & FSRQ \\
\hline
\end{tabular}

(Tegmark \& Efstathiou 1996; Scott \& White 1999). The quantity at right hand side in Eq. (2) is the usual Poisson shot-noise term (Peebles 1980; Tegmark \& Efstathiou 1996) and a further term, $\omega(I)^{2}$, adds to it if the clustering of sources is not 

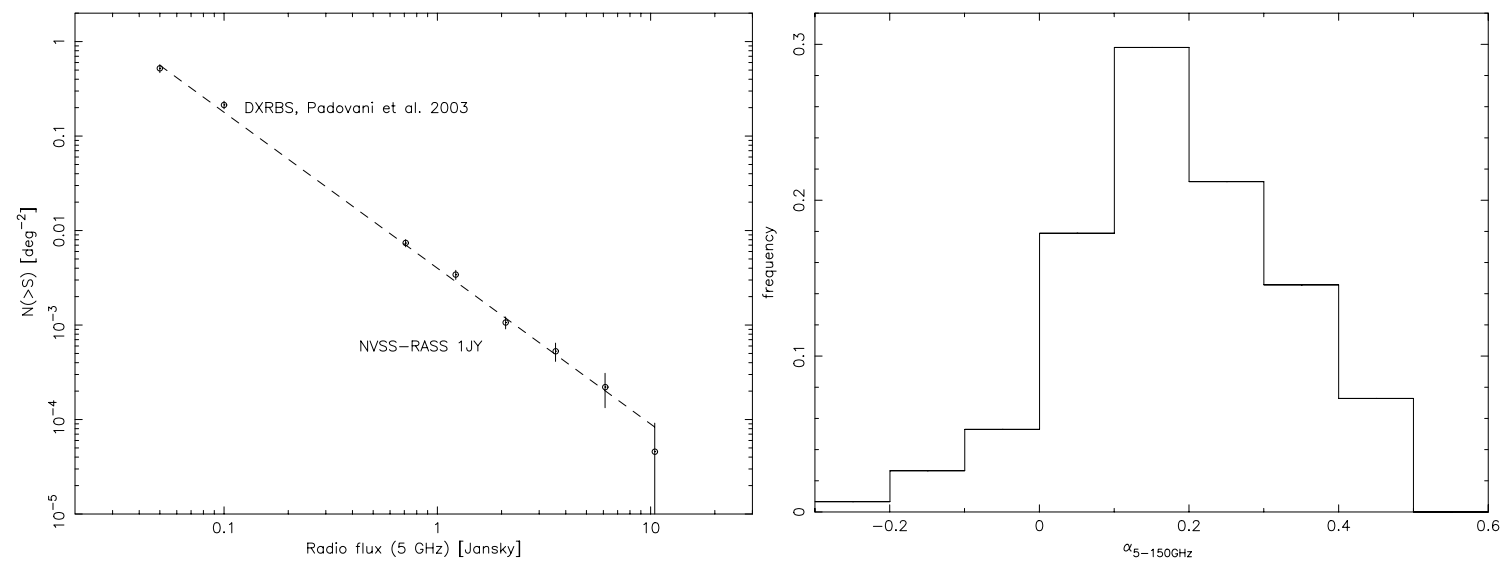

Fig. 3. Left: the $5 \mathrm{GHz}$ (integral) $\log N-\log S$ of Blazars built with data from the NVSS-RASSS 1 Jy survey and the DXRBS point at 0.1 and $0.05 \mathrm{Jy}$. The dashed line is a power-law with slope 1.65. Right: we show here the distribution of spectral slopes between $\mathrm{cm}$ and $\mathrm{mm}$ wavelengths from the sample of 135 Blazars of the NVSS-RASS 1 Jy survey with $\alpha<0.5$.

negligible (Scott \& White 1999). This last clustering term can be even a factor $\sim 10$ larger than the Poissonian term at large and intermediate angular scales, $\ell<1000$ (Scott \& White 1999).

For the Blazar population described by the $\log N-\log S$ given in Fig. 3 we found $C_{\ell, \text { Blazar }} \approx 48 \mathrm{Jy}^{2} \mathrm{sr}^{-1}$ at $41 \mathrm{GHz}$ and $C_{\ell, \text { Blazar }} \approx 46 \mathrm{Jy}^{2} \mathrm{sr}^{-1}$ at $94 \mathrm{GHz}$. Our results can be translated into temperature units, as is usual in CMB anisotropy studies, using the conversion between the isotropic black-body (Planckian) brightness $B_{0}(v)$ and the CMB temperature $T_{0}$ which can be written as

$$
\begin{aligned}
\frac{\partial B_{0}}{\partial T_{0}} & =\frac{k}{2}\left(\frac{k T_{0}}{h c}\right)^{2} \times\left[\frac{x^{2}}{\sinh (x / 2)}\right]^{2} \\
& =\left(\frac{24.8 \mathrm{Jy} \mathrm{sr}^{-1}}{\mu \mathrm{K}}\right) \times\left[\frac{x^{2}}{\sinh (x / 2)}\right]^{2},
\end{aligned}
$$

where $x \equiv h v / k T_{0}=v / 56.84 \mathrm{GHz}$ is the a-dimensional frequency given in terms of the Planck constant $h$, of the speed of light $c$ and of the Boltzmann constant $k$. For WMAP, we found $C_{\ell, \text { Blazar }} \approx 2.0 \times 10^{-2} \mu \mathrm{K}^{2}$ sr at $41 \mathrm{GHz}$ and $C_{\ell, \text { Blazar }} \approx$ $1.0 \times 10^{-3} \mu \mathrm{K}^{2}$ sr at $94 \mathrm{GHz}$. In Fig. 4 we plot the quantity $C_{\ell, \text { Blazar }} \ell(\ell+1) /(2 \pi)$ and we compare it to the CMB fluctuation power spectrum which best fits the available data. The solid line in Fig. 4 shows the contribution of Blazars to the CMB fluctuation spectrum in the WMAP Q channel at $41 \mathrm{GHz}$ as evaluated from the $\log N-\log S$ given in Eq. (1) which yields $C_{\ell, \text { Blazar }} \approx 2.0 \times 10^{-2} \mu \mathrm{K}^{2}$ sr. We stress here that the previous value is a definite lower limit for $C_{\ell, \text { Blazar }}$ since it neglects the contribution of steep-spectrum sources at low frequencies which flatten at these frequencies (41 and $94 \mathrm{GHz}$ ) and the effect of flux variability. For this reason we also show a more realistic case for the Blazar power spectrum by adding the contribution of radio sources with steep-spectrum at low radio frequencies which flatten at higher frequencies (the dashed line in Fig. 4). These sources likely provide a value of $C_{\ell, \text { Blazar }}$ which is higher by a factor $\sim 2$ (see also discussion in Taylor et al. 2003). The effect of spectral and flux variability also allows for an increase of the fluctuation level $C_{\ell \text {,Blazar }}$ because many Blazars which are below the sensitivity threshold of $\mathrm{CMB}$ experiments can be detected during flares. The Blazar flux variability at millimeter wavelengths may be very substantial (higher than the factors 3-10 on time scales of weeks to years seen at $\mathrm{cm}$ wavelengths) and could definitely increase the level of contamination of CMB maps when these are built over long integration periods. The dotted line in Fig. 4 shows the Blazar power spectrum when also this last contribution is taken into account.

To better quantify the level of contamination of the CMB power spectrum induced by Blazars we show in the right panel of Fig. 4 the ratio $C_{\ell \text {, Blazar }} / C_{\ell, \mathrm{CMB}}$ where $C_{\ell, \mathrm{CMB}}$ has been evaluated in a $\Lambda$ CDM cosmology with $\Omega_{m}=0.3, \Omega_{\Lambda}=0.65, \Omega_{b}=$ 0.05 which best fits the available data. It is evident from our results that, even in the case of the lower limit to $C_{\ell \text {, Blazar }}$ (solid curve in Fig. 4), the population of flat spectrum radio sources produces a power spectrum which amounts to $\sim 30 \%$ of the $\mathrm{CMB}$ power spectrum at $\ell \approx 500$ and reaches $\sim 70 \%$ of the $\mathrm{CMB}$ power spectrum at $\ell \approx 600$. In this case the Blazar power spectrum equals the $\mathrm{CMB}$ power spectrum at $\ell \sim 850$. In a more realistic case, the addition of steep-spectrum radio sources at low- $v$ which flatten at higher $v$ 's makes the contamination of the primordial CMB spectrum more significant. In fact, in this last case, the Blazar power spectrum (dashed curve in Fig. 4) reaches a level $\sim 50 \%$ at $\ell \approx 500$ and nearly reaches equality at $\ell \approx 530$. Inclusion of variability effects increases even more the contamination (dotted curve in Fig. 4) reaching a level of $\sim 90 \%$ at $\ell \approx 500$ and equalling the CMB power spectrum at $\ell \gtrsim 500$. Here we have not considered the statistical uncertainties of the CMB power spectrum data. These results show that Blazar-like radio sources introduce a non-negligible contamination in CMB maps and in the CMB power spectrum reconstruction, even in the lower-limit case of considering just the flat-spectrum sources at high frequencies. In this latter case, that the Blazar contamination of CMB maps decreases with increasing frequency and it becomes $\sim$ a few $\%$ at $\ell \lesssim 500$ for $v \gtrsim 90 \mathrm{GHz}$.

We also stress that the previous calculations are performed neglecting the clustering term $\omega(I)^{2}$ and thus they must be considered again as a lower limit to the realistic angular power spectrum contributed by the Blazars. Such a clustering term could produce even a factor $\sim 10$ amplification of the power 

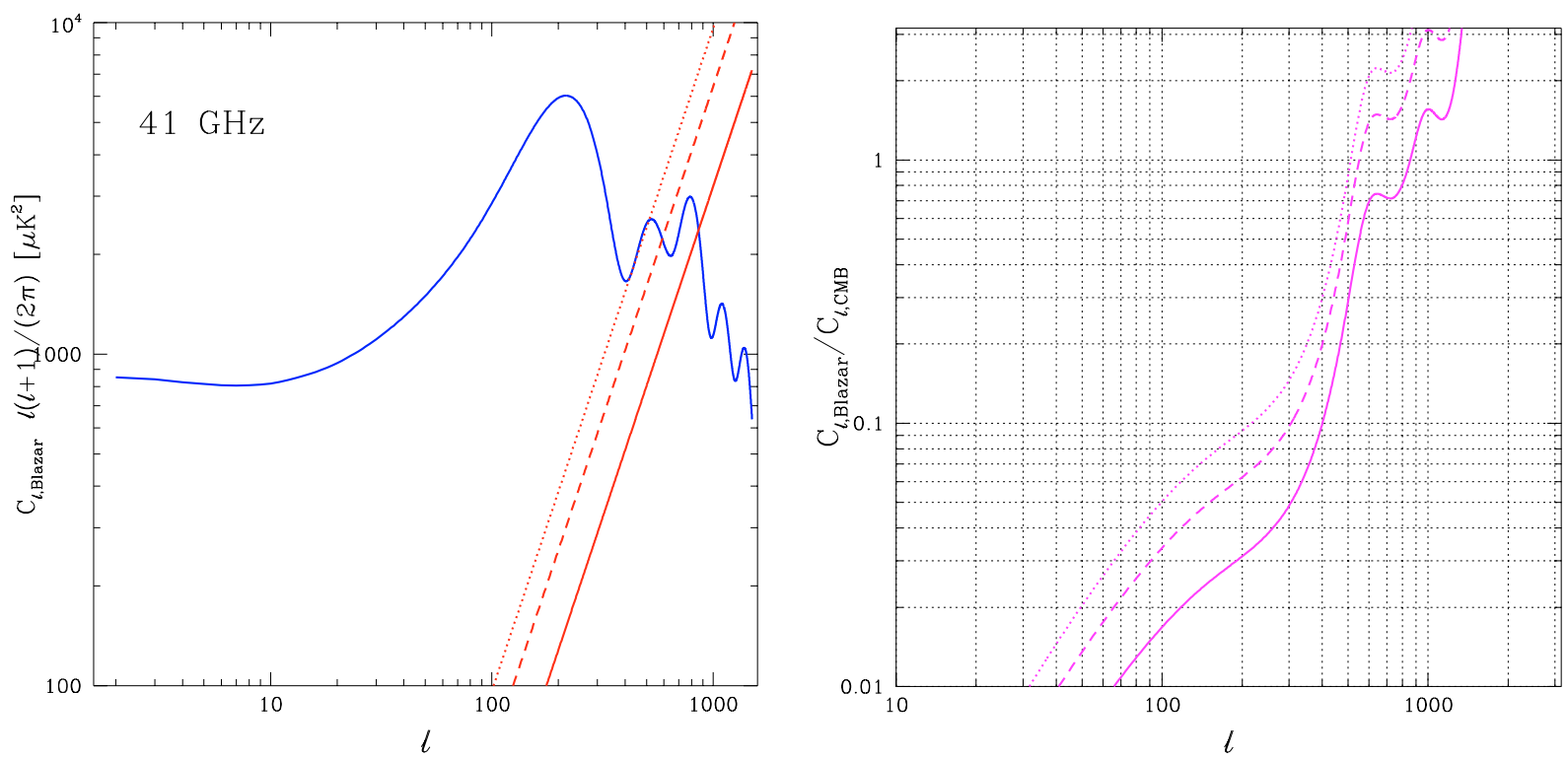

Fig. 4. Left: we show the contribution of Blazars to the CMB fluctuation spectrum in the WMAP Q channel at $41 \mathrm{GHz}$ as evaluated from the $\log N-\log S$ given in Sect. 4 (solid line). We also show the angular power spectrum for the Blazar population by adding an estimate of the possible contribution of radio sources with steep-spectrum at low radio frequencies which flatten at higher frequencies (dashed line). The dotted line includes also the effect of spectral and flux variability (see text for details). Although this additional contamination may be substantial a precise estimation can only be done through simultaneous high resolution observations at the same frequency. A typical CMB power spectrum evaluated in a $\Lambda \mathrm{CDM}$ cosmology with $\Omega_{m}=0.3, \Omega_{\Lambda}=0.65, \Omega_{b}=0.05$ which best fits the available data is shown for comparison. Right: we show here the ratio $C_{\ell, \text { Blazar }} / C_{\ell, \mathrm{CMB}}$ between the Blazar power spectra and the CMB power spectrum for $41 \mathrm{GHz}$.

spectrum at any $\ell<10^{3}$ (see, e.g., Scott \& White 1999). We will consider more specifically this additional contribution elsewhere (Giommi \& Colafrancesco in preparation).

It stems from the previous analysis, that the low- $\ell$ part of the reconstructed $\mathrm{CMB}$ power spectrum is heavily contaminated by Blazars even at $\ell \sim 400$, where the second and third acoustic peaks are expected in the best-fit cosmological model (i.e., a scenario with $\Omega_{m} \approx 0.3, \Omega_{\Lambda} \approx 0.65, \Omega_{b} \approx 0.05$ ). Thus, the non-negligible Blazar contamination of the CMB anisotropy spectrum at $\ell \gtrsim 400$ has evidently an impact on the derivation of the cosmological information which is contained in the CMB anisotropy spectrum. The second acoustic peak at $\ell \approx 500$ is considered as an incontrovertible evidence of inflationary sound waves (see, e.g., Hu \& White 1997) and its amplitude is mainly sensitive to the values of $\Omega_{b} h^{2}$ and $\Omega_{m} h^{2}$. Increasing $\Omega_{b} h^{2}$ has the effect of decreasing the amplitude of the second peak relative to the first and third peaks. A contamination of $\sim 30-90 \%$ at $\ell \approx 500$ would tend to decrease the power of the CMB spectrum at the position where the second peak is expected in a $\Lambda$ CDM scenario. This effect, by itself, would bias the quantities $\Omega_{b} h^{2}$ and/or $\Omega_{m} h^{2}$ toward high values. The third acoustic peak (and in principle all the acoustic peaks) is mainly depending on the amount of Dark Matter in the universe and its amplitude decreases for increasing values of $\Omega_{m} h^{2}$. The high level of contamination ( $\left.80-100 \%\right)$ of the CMB power spectrum at $\ell \approx 800$ as well as the intrinsic uncertainty of the data render the determination of the third acoustic peak problematic. The first acoustic peak at $\ell \approx 300$ is contaminated at a level $\sim 10 \%$ and hence the constraint on $\Omega_{0}$ set by CMB anisotropy data are little affected by the Blazar contamination, unless a substantial clustering of these sources can be considered as realistic. The present data do not provide strong insights on this last point and we will study it in more details in a forthcoming paper (Giommi \& Colafrancesco, in preparation).

Finally, we stress that the Blazar emission is significantly polarized at the level of $\sim 2-10 \%$ at radio and optical frequencies. In fact $96 \%$ of the WMAP sources which are included in the NVSS catalog are significantly polarized at $1.4 \mathrm{GHz}$ with typical percentage values ranging from 1 to $5 \%$ and in some cases reaching polarization levels well above $10 \%$. As a consequence of the non-negligible Blazar contamination of the CMB maps, the primordial polarization patterns could be heavily contaminated by these polarized sources. Hence, a significant contamination of CMB fluctuation maps and the non-negligible fraction of residual unresolved sky brightness will complicate the detection of primordial polarization fluctuations, as these are expected to be present only at a very low level, i.e. at a level $\sim 20-50$ lower than the primary CMB fluctuations (see, e.g., Hu \& White 1997).

\section{Conclusions}

In this paper we studied the contamination of CMB fluctuation maps induced by flat-spectrum extragalactic radio sources which shine in the frequency bands probed by BOOMERANG and WMAP. Blazars have in fact a typical spectrum which is similar to the CMB spectrum in the range $40-150 \mathrm{GHz}$. We predicted the level of brightness and of the induced CMB anisotropy using the most updated version of the Blazar $\log \mathrm{N}$ $\log S$. 
In view of the results presented in the previous sections, the main conclusion of this paper can be summarized as follows:

- Blazars (BL Lacs, Flat Spectrum Radio Quasars, and radio sources with Blazar-like characteristics) are by far the major point-like, bright source contaminant of CMB maps.

- We expect more than 200 Blazars to be present in the BOOMERANG $90 / 150 \mathrm{GHz}$ maps above the sensitivity limit. We already identified more than 50 of these sources on the basis of a detailed analysis of known and candidate Blazars. We have also found a very large number of Blazars (180 out of the 184 identified sources) in the WMAP bright source catalog.

- The importance of Blazars as contaminants of CMB fluctuation maps stems from the fact that their $\log N-\log S$ is steeper than that of flat spectrum radio sources previously used to estimate the contamination of CMB maps by extragalactic sources. The observed Blazar $\log N-\log S$ at $5 \mathrm{GHz}$ combined with the measured spectral slope distribution implies a $\log N-\log S$ at higher frequencies which is steeper than Euclidean at least down to $50 \mathrm{mJy}$ at all frequencies relevant for $\mathrm{CMB}$ experiments. We expect, in fact, $\sim 0.5$ sources per $\operatorname{deg}^{2}$ at $F \geq 50 \mathrm{mJy}$, quite independently of the frequency.

- We expect an induced fluctuation amplitude of the CMB map of order of $C_{\ell, \text { Blazar }} \approx 48 \mathrm{Jy}^{2} / \mathrm{sr}$ which corresponds to a temperature fluctuation of $\sim 0.02 \mu \mathrm{K}^{2}$ sr at $41 \mathrm{GHz}$. The corresponding Poissonian spectrum of the Blazar population equals the $\mathrm{CMB}$ power spectrum at $\ell \approx 850$, and contaminates it at levels $\sim 30 \%$ at $\ell \approx 500$ and $\sim 80 \%$ at $\ell \approx 800$.

- These estimates of $C_{\ell, \text { Blazar }}$ are a firm lower limit to the level of CMB fluctuation induced by the Blazar population. In fact, the value of the Poissonian term $C_{\ell, \text { Blazar }}$ is likely to be higher by a factor at least $\gtrsim 2-3$ considering both the Blazar variability and the contribution of steepspectrum sources at low frequencies, radio-galaxies and possibly starburst galaxies at lower fluxes. We also stress that allowance for an appropriate clustering term might enhance substantially the angular power spectrum of the point-like sources and hence increase the level of contamination of the CMB power spectrum.

- It is important to stress that Blazar variability increases with increasing frequency and, consequently, that flux variation at millimeter wavelengths may be very substantial, higher and possibly more frequent than the factors 3-10 on time scales of weeks to months seen at $\mathrm{cm}$ wavelengths. This definitely increases the level of contamination of CMB maps when these are built over long integration periods as in satellite experiments.

- As a consequence of the non-negligible Blazar contamination of the CMB maps, the primordial CMB polarization seems to be greatly affected by the presence of polarized Blazars as demonstrated by WMAP point sources (see Sect. 5). Thus, the significant contamination of $\mathrm{CMB}$ fluctuation maps and the non-negligible fraction of residual unresolved sky brightness will render difficult to detect primordial polarization fluctuations.
The many high-sensitivity and high-resolution CMB experiments planned for the near future will definitely face with the problems highlighted in this paper. In fact, we predict at least 1600 sources/sr at $90 \mathrm{GHz}$ with flux larger than $50 \mathrm{mJy}$ and a significant level of polarization which produce a brightness excess $\gtrsim 10 \mu \mathrm{K}$ in a pixel of 15 arcmin size.

We have shown that the dominant population of bright extragalactic sources that contaminate CMB maps are Blazars, a class of objects closely pointing their radio jet towards us and emitting strongly in the microwave region as well as over the entire e.m. spectrum. This implies that at faint fluxes we should find a much larger number of intermediate blazar-like sources (those pointing their jet at larger angles with respect to the observer and with probably a steeper spectrum at $\mathrm{cm}$ wavelenghts) that will heavily contaminate future high sensitivity CMB maps. These sources, however, can in principle be efficiently identified exploiting their peculiar SED by means of multi-frequency detection techniques, and then efficiently subtracted from CMB anisotropy maps.

One proven way of finding Blazars down to faint radio fluxes $(\sim \mathrm{mJy})$ is to use highly efficient multi-frequency selection techniques, similarly to the DXRBS survey which has an efficiency of $\sim 90 \%$ (Perlman et al. 1998; Landt et al. 2001), by cross correlating deep radio surveys (e.g., NVSS, FIRST etc.) with deep X-Ray data over the largest possible area of sky. However, to reach flux levels lower than $\sim 10 \mathrm{mJy}$, this approach requires an X-ray sensitivity of $\sim 10^{-15} \mathrm{erg} \mathrm{cm}^{-2} \mathrm{~s}^{-1}$. Such a sensitivity is reached in XMM-Newton and Chandra Xray images only for a very limited area of sky. In conclusion, it is crucial to determine the level of Blazar contamination in precision $\mathrm{CMB}$ experiments in order to separate the contaminating foreground from the primordial CMB fluctuations and to provide a reliable determination of the cosmological parameters.

Acknowledgements. The authors are grateful to E. Massaro for useful discussions, to P. Padovani for providing DBRBS results in advance of publication, and to M. Perri, E. Cavazzuti and S. Piranomonte for their help in the analysis of part of the NVSS-RASS 1JY data. We also thank N. Panagia for useful comments on a preliminary version of the paper. This research has made use of data from the ASI Science Data Center and from the NASA/IPAC Extragalactic Database (NED). We thank the referee for several useful suggestions that allowed us to improve the presentation of our paper.

\section{References}

Bade, N., Beckmann, V., Douglas, N. G., et al. 1998, A\&A, 334, 450 Bennett, C. L., Bay, M., Halpern, M., et al. 2003a, ApJ, 583, 1

Bennett, C. L., Hill, R. S., Hinshaw, G., et al. 2003b, ApJS, 148, 97

Blandford, R. D., \& Rees, M. J. 1978, in Pittsburgh Conf. on BL Lac objects, Pittsburgh, Pa., April 24-26, Proc. (A79-30026 11-90) Pittsburgh, Pa., University of Pittsburgh, 328; Discussion, 341. NATO-supported research

Bond, J., Efstathiou, G., \& Tegmark, M. 1997, MNRAS, 291, 33

Coble, K., Ade, P. A. R., Bock, J. J., et al. 2003, ApJS, submitted [astro-ph/0301599]

De Bernardis, P., Ade, P. A. R., Bock, J. J., et al. 2000, Nature, 404, 955 
Giommi, P., Menna, M. T., \& Padovani, P. 1999, MNRAS, 310, 465

Giommi, P., Capalbi, M., Fiocchi, M., et al. 2002a, in Proc. of the workshop: Blazar Astrophysics with BeppoSAX and Other Observatories, ed. P. Giommi, E. Massaro, \& G. Palumbo (ASI special publication), 63

Giommi, P., Perri, M., Landt, H., \& Perlman, E. 2002b, in Proc. of the workshop: Blazar Astrophysics with BeppoSAX and Other Observatories, ed. P. Giommi, E. Massaro, \& G. Palumbo (ASI special publication), 133

Giommi, P., Perri, M., Piranomonte, S., \& Padovani, P. 2002c, in Proc. of the workshop: Blazar Astrophysics with BeppoSAX and Other Observatories, ed. P. Giommi, E. Massaro, \& G. Palumbo (ASI special publication), 123

Grainge, K., Carreira, P., Kieran, C., et al. 2003, MNRAS, 341, L23

Halverson, N. W., Leitch, E. M., Pryke, C., et al. 2002, ApJ, 568, 38

Hanany, S., Ade, P. A. R., Balbi, A., et al. 2000, ApJ, 545, L5

Hasinger, G. 2000, in ISO Surveys of a Dusty Universe, Proc. of a Ringberg Workshop Held at Ringberg Castle, Tegernsee, Germany, 8-12 November 1999, ed. D. Lemke, M. Stickel, \& K. Wilke, Lecture Notes in Physics, 548, 423

Hu, W., \& White, M. 1997, New Astron., 2, 323

Hughes, P. A., Aller, H. D., \& Aller, M. F. 1992, ApJ, 396, 469

Jungman, G., Kamionkowski, M., Kosowski, A., \& Spergel, D. 1996, Phys. Rev. D, 54, 1332

Landt, H., Padovani, P., Perlman, E. S., et al. 2001, MNRAS, 323, 757

Laurent-Muehleisen, S. A., Kollgaard, R. I., Ciardullo, R., et al. 1998, ApJS, 118, 127
Myers, S. T., Contaldi, C. R., Bond, J. R., et al. 2003, ApJ, 591, 575

Padovani, P. 2002, in Proc. of the workshop: Blazar Astrophysics with BeppoSAX and Other Observatories, ed. P. Giommi, E. Massaro, \& G. Palumbo (ASI special publication), 101

Padovani, P., et al. 2003, in preparation

Pearson, T. J., Mason, B. S., Readhead, A. C. S., et al. 2003, ApJ, 591, 566

Peebles, P. J. E. 1980, The Large-Scale Structure of the Universe (Princeton: Princeton Univ. Press)

Perlman, E. S., Padovani, P., Giommi, et al. 1998, AJ, 115, 1253

Perri, M., Giommi, P., Piranomonte, S., \& Padovani, P. 2002, in Proc. of the workshop: Blazar Astrophysics with BeppoSAX and Other Observatories, ed. P. Giommi, E. Massaro, \& G. Palumbo (ASI special publication), 119

Pierpaoli, E. 2003, ApJ, 589, 58

Rector, T. A., Stocke, J. T., Perlman, E. S., et al. 2000, AJ, 120, 1626

Refregier, A., Spergel, D. N., \& Herbig, T. 2000, ApJ, 531, 31

Scott, D., \& White, M. 1999, A\&A, 346, 1

Smoot, G. F., Bennett, C. L., Kogut, A., et al. 1992, ApJ, 396, L1

Stickel, M., Padovani, P., Urry, M., et al. 1991, ApJ, 374, 431

Taylor, A. C., Carreira, P., Kieran, C., et al. 2003, MNRAS, 341, 1066

Tegmark, M., \& Efstathiou, G. 1996, MNRAS, 281, 1297

Toffolatti, L., Argueso, G. F., deZotti, G., et al. 1998, MNRAS, 297, 117

Urry, C. M., \& Padovani, P. 1995, Publ. Astr. Soc. Pacific, 107, 803

Wall, J. V., \& Peacock, J. A. 1985, MNRAS, 216, 173 